\title{
Strategy enhancement of serial motor skill acquisition
}

\author{
ROBERT N. SINGER, FRANKLIN HAGENBECK, and RICHARD F. GERSON \\ Florida State University, Tallahassee, Florida 32306
}

\begin{abstract}
Male high school students $(\mathbf{N}=75)$ were randomly assigned to five groups in order to learn a curvilinear repositioning task. Five conditions were compared: (1) analogous imagery, (2) relevant labeling, (3) irrelevant labeling, (4) kinesthetic, and (5) control. Six positions, each different for each trial, had to be learned in sequence for 10 trials. An analysis of absolute and variable error scores indicated the general superiority of the imagery group over the other groups. Analysis of the percentage of correct responses at each criterion position demonstrated the effectiveness of imagery and relevant labeling.
\end{abstract}

Persons required to learn a serial motor skill must acquire the components in a sequential order. These individuals tend to exhibit a "bow-shaped" curve that is indicative of the accuracy of recalling a given item in the sequence, based on the ordinal position of that item. Usually, material presented either early or late in the sequence is recalled most accurately, whereas the components that are presented during the middle of the list are recalled with the greatest number of errors. These results have been explained within a dual-stage theory of memory, as earlier presented items are presumably rehearsed more and therefore retrieved from the longterm store and last-presented items are retrieved from the short-term store. Retrieval of middle items shows the greatest errors because these items are supposedly in a state of incomplete transfer from short-term store to long-term store (Glanzer, 1972; Glanzer \& Koppenaal, 1977). Likewise, retroactive and proactive interference can occur with these middle items.

The effect of strategy usage on information in memory is to lead to a greater organization of that material, which increases the amount of information that can be stored. Subsequently, higher overt performance levels will be evidenced. The learning of a serial motor task will be even further enhanced if the organizational structure accounted for the relative position of items in a sequence. This would negate any possible interference effects (Magill, 1976), as the cognitive boundaries established by the organization of the information should protect items in those groupings from interference by any other items (Harcum, 1975).

Based on the positive effects of strategy usage in verbal learning (see Flavell \& Wellman, 1977, for a

This study was supported in part under Contract MDA90377-C-0200, received by the first author from the Defense Advanced Research Projects Agency. Requests for reprints should be addressed to Robert N. Singer, Movement Science and Physical Education Department, Florida State University, Tallahassee, Florida 32306. review) and the relative lack of comparable studies in the motor skills area, this investigation was designed to assess the effectiveness of various strategies in the learning of a serial motor skill. An effective strategy should improve performance on a serial motor task at the initial, middle, and terminal positions of a sequence by enhancing the memory representations and thus, the storage and retrieval of the items. Therefore, it was hypothesized in this study that performance under strategy instructions should exceed performance in a control condition.

\section{METHOD}

Subjects

Subjects were 75 right-handed 11 th- and 12 th-grade male volunteers (mean $=17.30$ years) from a private church-related school in Tallahassee, Florida. They were randomly blocked into five groups.

\section{Apparatus}

The task was a curvilinear repositioning apparatus composed of a $36 \times 64 \mathrm{~cm}$ flat metal base and a $25-\mathrm{cm}$ metal pointer affixed to the base on a ball bearing mechanism such that the pointer pivoted through a range of $200 \mathrm{deg}$ in the horizontal plane. A handle, $8.25 \mathrm{~cm}$ high, was attached vertically to the pointer to facilitate movement of the pointer.

\section{Procedure}

A 5 by 6 (strategies by criterion positions) randomizedblocks design provided the appropriate information in this study. The five strategy groups were (1) an analogous imagery group (IM), which during rehearsal was provided with an analogous image of each movement and each criterion position by the experimenter's positioning of the black minute hand on a clockface, (2) a relevant labeling group (RL), which was provided with labels in degrees for each criterion position by the experimenter, (3) an irrelevant labeling group (IR), which counted forward by ones from the start position to each criterion position and labeled each one numerically and consecutively, (4) a kinesthetic group (KS), which was instructed to rely solely on the "feel" of the movement and the "feel" of the criterion positions, and (5) a control group, which counted backward by threes from a three-digit number to prevent the use of a self-generated covert strategy. Criterion positions consisted of six locations that were randomly selected between the 
15 - $\mathrm{min}$ and 45 -min markings $(1 \mathrm{~min}=6 \mathrm{deg})$ on a clockface, thus encompassing the 0 - to $180 \mathrm{deg}$ range on the test apparatus.

A complete testing session consisted of 10 serial positioning trials administered in two blocks of five trials each with $5 \mathrm{~min}$ rest between the blocks. There were six randomly selected criterion positions for each of the 10 sequences. Each serial positioning trial consisted of three practice attempts that were followed immediately by a recall test. The first practice attempt required a subject to move the pointer sequentially to each criterion position, which was marked by a peg inserted in the face of the apparatus. The movement of the pointer was automatically stopped by the pegs at each criterion position. The second and third practice attempts were condurted without the pegs. The subject moved the pointer and sequentially stopped at what was estimated to be each criterion position. At each stop, the experimenter provided immediate knowledge of results as to degrees off target. Following the recall test, an intertrial rest of $30 \mathrm{sec}$ was given.

\section{RESULTS}

An analysis of absolute error (AE) provided significant differences for all main effects and all interactions. The main effect for strategy was significant $[F(4,840)=$ $48.99, \mathrm{p}<.001$ ] . Subsequent post hoc analysis showed that the IM group was significantly more accurate than any of the four remaining strategy groups, which were not different from each other. The main effect for position was significant $[F(5,840)=3.09, p<.01]$. Further analysis showed that all groups were more accurate at Position 3 than at either Position 2 or 5. The Strategy by Position interaction was significant $[F(20,840)=7.18, p<.001]$ and is illustrated in Figure 1.

Analysis of variable error (VE) indicated that there were differences for both main effects and the firstorder interaction. The main effect for strategy was significant $[F(4,840)=56.02, p<.001]$. The IM group forgot less than the remaining four strategy groups, and the RL group forgot less than the IR, the KS, or the control group. The main effect for position was significant $[F(4,840)=2.71, p<.05]$. Performances at Positions 4, 5 , and 6 were all poorer than those at Position 1, but they were not significantly different from each other.

A serial position curve was graphed for each group by plotting the percentage of correct responses at each criterion position. A correct response was considered to be $\pm 3 \mathrm{deg}$ from the criterion position. This measurement

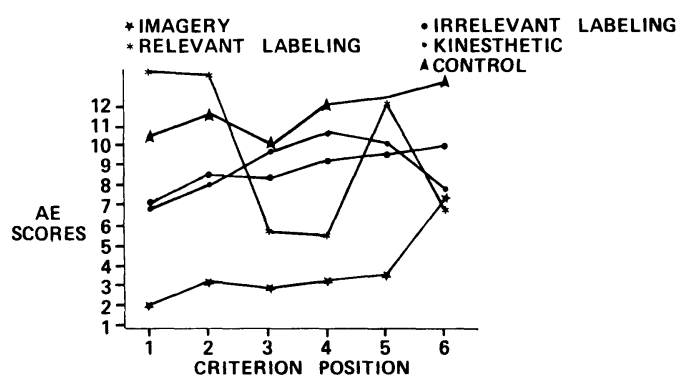

Figure 1. Strategy by Position interaction for AE.

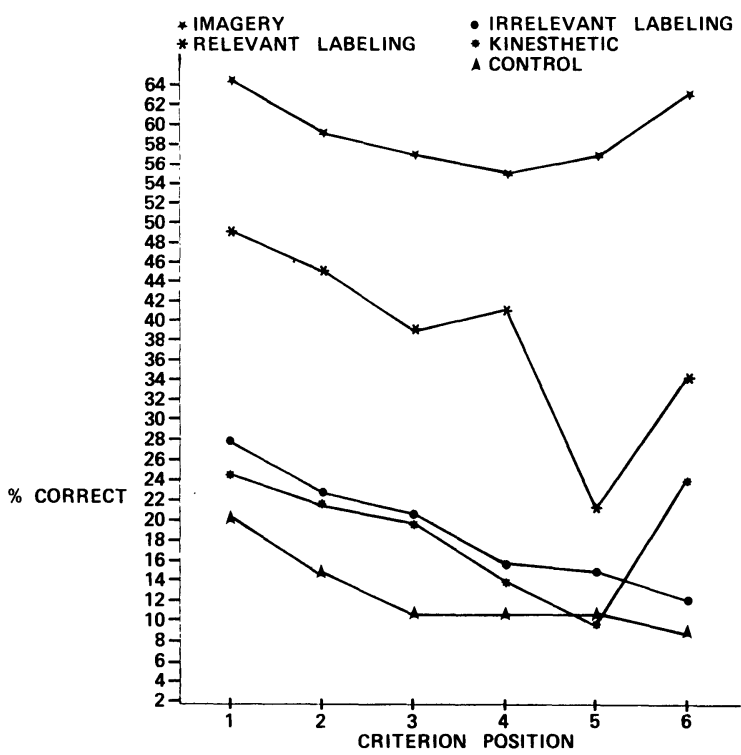

Figure 2. Strategy by Position interaction for percentage of correct responses.

was similar to the "all-or-none" response scores employed in verbal learning studies. A U-shaped curve with a strong primacy effect and a slight recency effect occurred for the IM, RL, and KS groups, whereas a linear trend showing a negative recency effect occurred for the IR and control groups.

The analysis of variance performed on the percentage of correct responses showed a main effect for strategy $[F(4,840)=298.90, p<.001]$ that comprised $81 \%$ of the shared variance. The IM group had a higher percentage of correct responses than the four remaining groups, and the RL group had a higher percentage of correct responses than did the IR, KS, and control groups. The main effect for position was also significant $[F(5,840)=47.34, p<.001]$. More incorrect responses were made at Positions 4 and 5 than at Position 1.

The interaction of Strategy by Position (see Figure 2) was significant $[F(20,840)=2.82, p<.01]$. At Positions 14, the IM group had a higher percentage of correct responses than did the IR, KS, and control groups, and at Positions 5 and 6 , the IM group evidenced a higher percentage of correct responses than any of the four remaining strategy groups. Similarly, the RL group had a greater percentage of correct responses at Positions 1-4 than did the IR, KS, and control groups, which were not significantly different from each other.

\section{DISCUSSION}

In general, the implementation of an imagery mnemonic led to greater accuracy and response consistency at recall than did any other type of strategy. Certain strategies elevated the response scores in the middle positions of the curve. The best strategies for the serial acquisition of the task were imagery and relevant labeling, both of which resulted in greater percentages of correct responses of the middle-position targets than did the 
other strategy groups. The only deviation from the pattern was for the RL group, which evidenced a low percentage of correct responses at Position 5. This may have been due to an inefficient coding of the items in this position, which was possible from interference from previous responses. However, the general conclusion was substantiated by the superior performance of the RL group at Positions 14 when compared with the IR, $\mathrm{KS}$, and control groups.

Motor skill acquisition through strategy usage has been less studied than verbal learning, so there remains a degree of uncertainty as to which strategies, introduced in what manner, will lead to improved skill. For serial motor skills to be acquired and performed well, a learner must invoke cognitive strategies to organize information. The degree of involvement of learners in this process will be demonstrated by performance levels on a recall test. Superior performance would imply greater organization. It can be concluded from the present results that cognitive strategies are much more involved in the acquisition of complex motor behaviors than has heretofore been realized and that various strategies differentially affect skill acquisition.

\section{REFERENCES}

Flavell, J. H., \& Wellman, H. M. Metamemory. In R. V. Kail \& J. W. Hagen (Eds.), Perspectives on the development of memory and cognition. Hillsdale, N.J: Erlbaum, 1977.

Glanze R, M. Storage mechanisms in recall. In G. H. Bower (Ed.), The psychology of learning and motivation: Advances in research and theory (Vol. 5). New York: Academic Press, 1972.

Glanzer, M., \& Koppenaal, L. The effect of encoding tasks on free recall: Stages and levels. Journal of Verbal Learning and Verbal Behavior, 1977, 16, 21-28.

Harcum, E. R. Serial learning and paralearning: Control processes in serial acquisition. New York: Wiley, 1975.

MAGILL, R. A. Order of acquisition of the parts of a serial motor task. Research Quarterly, 1976, 47, 134-142.

(Received for publication July 13, 1981.) 